Assertive community outreach can be in an addiction clinic, postnatal ward, mental health centre, psychiatric ward, outpatient clinic, homeless hostel or the client's home.

Time allocation for outreach and priority appointment-based care was 8 and 4 hours per week respectively. Care in both pathways was provided by senior doctors. Content of care was similar but facility for provision of gynaecological care including cervical smears and investigations for abnormal uterine bleeding e.g. pelvic ultrasound scans and endometrial biopsies were only available in the mainstream clinic setting at CSHC.

Result. From May 2016 to December 2020 SHRINE received 1367 referrals from 125+ teams. We offered 1591 first or follow-up appointments of which 1369 (86\%) were attended. A total of $1153(84 \%)$ of our patient contacts occurred in the outreach setting where 93\% the appointments were attended. Of the 358 appointments at CSHC $316(60 \%)$ were attended.

Conclusion. Making clinic access as simple and convenient as possible is not a sufficient strategy to meet the SRH needs of marginalised people. To enable them to realise their human right to sexual and reproductive health we need to leave our clinics and meet our clients where they are. A combined model of outreach and priority access clinic pathways is essential for provision of SRH care for people with mental illness.

\section{Working with catatonia: a qualitative exploration of inpatient team emotional responses}

Emma Salter ${ }^{1 \star}$, Linda Pow ${ }^{1}$, Emma Stacey ${ }^{1}$, Victoria Stephens ${ }^{1}$, Paul Beckley ${ }^{1}$, Jenna Oliphant ${ }^{1}$, Lottie Heimes ${ }^{2}$ and Courtney Foster ${ }^{2}$

${ }^{1}$ Somerset Partnership NHS Foundation Trust and ${ }^{2}$ Somerset NHS Foundation Trust

${ }^{\star}$ Corresponding author.

doi: 10.1192/bjo.2021.909

Aims. Child and adolescent mental health (CAMHS) wards treat patients with variable presentations. During diagnosis and treatment, psychiatric professionals use structured criteria, but also honed awareness of countertransference. Unacknowledged emotional responses can produce powerful dynamics and impact patient care.

Limited information exists on possible emotional responses and team dynamics when working with catatonia.

This project aimed to establish common themes relating to staff felt-experience of working with a specific case of catatonia on a Child and Adolescent Mental Health (CAMHS) ward. A secondary aim was to establish potential areas for future training and service improvement.

Method. Trust Research and Development department approved this work. Inpatient professionals working with the specified patient during admission were eligible. Participants were invited via email and face-face discussion with one of the authors. Participants, patient and mother provided written consent.

A questionnaire was created and disseminated via email to eligible staff $(\mathrm{n}=33) .27$ questions asked individuals to rate responses on Likert scales, plus space for further comments. Questions involved emotional responses to different catatonic states, feelings towards self, patient, colleagues and plans. Descriptive analysis was completed on this anonymised data.

Qualitative data were gathered via 1-hour recorded focus group, led by a systemic psychotherapist and psychologist. The session was transcribed anonymously. Two clinicians, using Thematic Analysis, reviewed the transcript independently.
Result. 16 (48.5\%) questionnaires were completed. Participants felt negatively about themselves and colleagues more frequently than about the patient. Participants felt positively about themselves less frequently than about colleagues and the patient. Participants identified with more feelings during immobile patient states than lucid states. During immobile states, participants identified with abusive, guilt, hopeless and neglectful responses; during lucid states, with helpful, caring, happy responses

Eight (50\%) participants felt they sometimes did not understand their feelings towards colleagues/plans. Nine (57\%) participants felt they sometimes did not understand their feelings towards themselves. Ten (66\%) participants felt they sometimes did not understand their feelings towards the patient.

Ten $(62.5 \%)$ participants felt confused by their emotions at least some of the time. Two (12.5\%) frequently felt confused by their emotions.

Four participants attended the focus group. Themes included confusion, internal and team conflict.

Conclusion. Working with catatonia involved confusion and team splitting. Staff conflict between plans and morals resulted in painful emotions. Prompt psycho-education within teams working with uncommon presentations was identified as a focus for improvement. The authors plan to explore possible avenues for future teaching, learning and team support.

\section{Transition from child and adolescent mental health} services to adult mental health services

Tania Saour

Central North West and West London Mental Health Trust

doi: 10.1192/bjo.2021.910

Background. The prevalence and recognition of mental health conditions in young people is growing. Around 50\% of lifetime mental illness (except dementia) begins by the age of 14 . Around $75 \%$ of adults requiring secondary mental health services developed problems prior to 18 .

The TRACK study of young people's transitions from CAMHS to AMHS has found that up to a third of teenagers are lost from care during transition and a further third experience an interruption in their care.

A CQUIN for Transition has concluded that young children should have a transition plan 6 months before they turn 18 .

Method. All young people aged 17 and a half years old were included in the data collection for this audit. Clinical information was reviewed using the West London RIO computer system. While reviewing the clinical documentation I was recording whether:

Transitional plans had been discussed with the young person.

If yes, what were they?

Had a referral been made to the appropriate service?

Result. There were 180 open cases to the Hounslow Adolescent Team. 35 cases were over 18 :

At least 16 of these cases needed to be closed as no intervention was being provided.

14 cases had an unclear plan.

Of the 25 cases aged between 17.5 and 18 years of age transitional plans were:

Transition was discussed in 11 cases $(44 \%)$. This meant that transitional plans were not discussed in 56\% of young people.

Of these 11 cases 7 referrals were completed. (28\%)

Conclusion. The lack of consistent protocols for transition remains a significant barrier to health care provided to young people.

Transitional planning needs to take place in an effective and timely manner to ensure continued patient centred care. 
Transitional discussions to be made a regular agenda item at team meetings.

Care co-ordinator to be informed and reminded that transitional plans need to be explored with young people.

Following a re-audit of this data 6 months on $100 \%$ of cases over the age of 18 were closed and transition was discussed in the remaining $56 \%$.

\section{Where is my sample? Investigating pre-analytical pathology sampling errors in a psychiatric hospital}

\author{
Emma Scholes-Pearson ${ }^{1 \star}$ and Nieves Mercadillo ${ }^{2}$ \\ ${ }^{1}$ Warrington and Halton Teaching hospitals and ${ }^{2}$ North West \\ Boroughs Healthcare NHS Foundation Trust \\ ${ }^{*}$ Corresponding author.
}

doi: 10.1192/bjo.2021.911

Aims. Errors in the pathology sampling process can be costly for all stakeholders in any clinical setting; however, this process is often overlooked within psychiatry. Over the space of just a few short months at Hollins Park Psychiatric Hospital (HPH) such errors were reported to be numerous with staff raising multiple issues relating to the pathology sampling process. These issues often had a negative impact on patient care, leading to outcomes such as as re-bleeding of patients and delays in interventions. Here, we aim to identify the predominant sources of error in this process and suggest possible improvements to minimise these errors in the future. Method. Initially, we mapped and analysed each step of the sampling process as it is currently performed in order to identify areas of possible improvement. We then distributed questionnaires to all junior doctors - who are responsible for the handling of samples within the clinical setting - in order to establish error type and frequency. Questions also assessed individual confidence and familiarity with the sampling process.

Result. When mapping the sampling process, we identified all key steps required when sending samples from $\mathrm{HPH}$ to Warrington and Halton Hospitals laboratory. This included one pathway for sending routine bloods, and one pathway for urgent bloods. The process for sending routine bloods required more steps and ultimately took longer for samples to reach the laboratory - as expected. Of the issues identified during mapping of the pre-analytical phase, a majority of $77.7 \%$ of clinicians reported samples had gone missing or were unreported - with the reasons for this being undetermined in most cases - and 55.5\% reported their samples never reached the lab. While on the whole participants were comfortable with the steps involved in sending samples to the laboratory, $77.7 \%$ were not aware of the requirement to log samples as they were being sent. Conclusion. The reasons underlying errors in the sampling process at HPH were multifactorial and included a lack of clinician familiarity with correct procedure, poor sample recording/tracking and lengthy transit times between the patient and laboratory. Here we outline some simple evidence-based recommendations (including education of staff and improved tracking through an electronic requesting system) to help reduce errors and streamline the sampling process in the hopes of improving both efficiency and accuracy, reducing the financial and clinical impact.

\section{High dose antipsychotic treatment monitoring audit}

Jake Scott ${ }^{\star}$ and Jose Belda

Sussex Partnership NHS Foundation Trust

${ }^{\star}$ Corresponding author.

doi: 10.1192/bjo.2021.912
Aims. To quantify how many patients were prescribed high dose antipsychotic treatment (HDAT) and establish whether guidance for monitoring HDAT was being followed in an Assertive Outreach Team.

Background. Severe mental health disorders are associated with significant premature mortality, predominantly due to physical health conditions. Antipsychotic medications are associated with side effects, including metabolic syndrome and QT prolongation, which increase the risk of serious physical illness. HDAT is defined as when the total dose of antipsychotics prescribed exceeds $100 \%$ of the maximum BNF dose, if each dose is expressed a percentage of its maximum dose. There is limited evidence of clinical benefit with HDAT but an increased risk of side effects. Patients prescribed HDAT should therefore be monitored for side effects and clinical benefit. Sussex Partnership NHS Foundation Trust developed a form specifically for this purpose, to be completed in addition to a physical health assessment.

Method. All patients on caseload were audited using the electronic notes. Current inpatients were excluded, as inpatient HDAT monitoring forms are attached to paper drug charts and therefore were not available for review.

Result. A total of 61 patients were audited. Nine were excluded due to being inpatients. 16 were on community treatment orders and 26 were prescribed a long-acting antipsychotic injection. 10 were prescribed clozapine. The median number of medications prescribed was one. Four patients were prescribed HDAT ranging from $117-150 \%$ of the maximum BNF dose. Of these four, one had a HDAT form but this was out of date. 39 of 52 (75\%) patients audited had had a physical health assessment in the past 12 months. Two of the 13 missing a physical health assessment were on HDAT.

Conclusion. Physical health monitoring should be carried out for all patients on antipsychotics, but is particularly important for patients on HDAT. This audit identified a problem in both general physical health checks and HDAT monitoring. On discussion with the multi-disciplinary team a number of barriers to appropriate physical health monitoring were identified. There was a lack of awareness within the multi-disciplinary team that patients were receiving HDAT and regarding the implications for side effects. A reliable system to highlight the need for physical health checks was also missing and the team did not have sufficient equipment to perform the necessary checks. Identifying these barriers should enable improvements in physical health and HDAT monitoring which can be re-audited.

\section{Psychiatric liaison team memory pathway: does it achieve the standards set out in NICE clinical guideline 97 ?}

Manouri Senaratne ${ }^{1 \star}$, Afshan Jabeen ${ }^{1}$, Alisha Hyams ${ }^{1}$, Carol Deary ${ }^{1}$ and Sarah Stathers ${ }^{2}$

${ }^{1}$ South West Yorkshire Partnership NHS Foundation Trust and

${ }^{2}$ South West Yorkshire Partnership NHS Foundation Trust; Cumbria, Northumbria and Tees Valley NHS Foundation Trust ${ }^{\star}$ Corresponding author.

doi: 10.1192/bjo.2021.913

Aims. Early assessment, diagnosis and management for people living with dementia is essential, both for the patient and their carers. We recognised delays in established local pathways when patients had unplanned acute hospital admissions preventing them from attending memory diagnostic appointments. The Psychiatric Liaison Team (PLT) Memory Pathway was introduced 